\title{
On Bi-Decompositions of Logic Functions
}

\author{
Tsutomu Sasao \\ Department of Computer Science \\ and Electronics \\ Kyushu Institute of Technology \\ Iizuka 820, Japan
}

\author{
Jon T. Butler \\ Department of Electrical and \\ Computer Engineering \\ Naval Postgraduate School \\ Monterey, CA 93943-5121, U.S.A.
}

\begin{abstract}
A logic function $f$ has a disjoint bi-decomposition iff $f$ can be represented as $f=h\left(g_{1}\left(X_{1}\right), g_{2}\left(X_{2}\right)\right)$, where $X_{1}$ and $X_{2}$ are disjoint set of variables, and $h$ is an arbitrary two-variable logic fuction. $f$ has a non-disjoint bidecomposition iff $f$ can be represented as $f\left(X_{1}, X_{2}, x\right)=$ $h\left(g_{1}\left(X_{1}, x\right), g_{2}\left(X_{2}, x\right)\right)$, where $x$ is the common variable. In this paper, we show a fast method to find bidecompositions. Also, we enumerate the number of functions having bi-decompositions.
\end{abstract}

\section{Introduction}

Functional decomposition is a basic technique to realize economical networks. If the function $f$ is represented as $f\left(X_{1}, X_{2}\right)=h\left(g\left(X_{1}\right), X_{2}\right)$, then $f$ can be realized by the network shown in Fig. 1.1. To find such a decomposition,

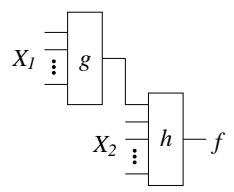

Figure 1.1: A Figure 1.2: A simple disjoint disjoint bi-decomdecomposition. position.

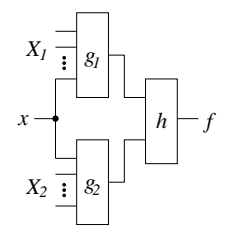

Figure 1.3: A non-disjoint bidecomposition. a decomposition chart with $2^{n_{1}}$ columns and $2^{n_{2}}$ rows are used, where $n_{i}$ is the number of variables in $X_{i}(i=1,2)$. When $n$ is large, the decomposition chart is too large to build. Recently, a method using BDDs has been developed [13]. This greatly reduces memory requirements and computation time. However, it is still time consuming, since we have to check all the $\left(\begin{array}{c}n_{1}+n_{2} \\ n_{1}\end{array}\right)$ partitions of $n=n_{1}+n_{2}$. In this paper, we consider bi-decompositions of logic functions, a restricted class of functional decompositions that have the form $f\left(X_{1}, X_{2}\right)=h\left(g_{1}\left(X_{1}\right), g_{2}\left(X_{2}\right)\right)$. Fig. 1.2 shows the realization of this decomposition.

The reasons we consider bi-decompositions are as follows:

1) If $f$ has no bi-decomposition, then the computation time is quite small.
2) Some programmable logic devices have two-input logic elements in the outputs.

3) If $f$ has a bi-decomposition, then the optimization of the expression is relatively easy.

A resticted class of bi-decompositions has been considered by [8]. The goals of this paper are

1) Present a fast method for finding bi-decompositions.

2) Enumerate the functions that have bi-decompositions.

Most of the proofs are omitted. They can be available from authors.

\section{Disjoint Bi-Decomposition}

Definition 2.1 Let $X=\left(X_{1}, X_{2}\right)$ be a partition of the variables. A logic function $f$ has a disjoint bidecomposition iff $f$ can be represented as $f\left(X_{1}, X_{2}\right)=$ $h\left(g_{1}\left(X_{1}\right), g_{2}\left(X_{2}\right)\right)$, where $h$ is any two-variable logic function.

If $f$ has a disjoint bi-decomposition, then $f$ can be realized by the network shown in Fig. 1.2.

Definition 2.2 Let $X=\left(X_{1}, X_{2}\right)$ be a partition of the variables. Let $n_{1}$ and $n_{2}$ be the number of variables in $X_{1}$ and $X_{2}$, respectively. A decomposition chart of the function $f$ for a partition $\left(X_{1}, X_{2}\right)$ consists of $2^{n_{1}}$ columns and $2^{n_{2}}$ rows of $0 s$ and $1 \mathrm{~s}$. The $2^{n_{1}}$ distinct binary numbers for $X_{1}$ are listed across the top, and the $2^{n_{2}}$ distinct binary numbers for $X_{2}$ are listed down the side. The entry for the chart corresponds to the value of $f\left(X_{1}, X_{2}\right)$.

Example 2.1 Two decomposition charts for the function $f\left(x_{1}, x_{2}, x_{3}, x_{4}\right)=x_{1} x_{2} \oplus x_{3} x_{4}$ are shown in Fig. 2.1 (a) and $(b)$.

Note that the decomposition chart is similar to the Karnaugh map with a different ordering for the cell locations.

Definition 2.3 The number of distinct column (row) patterns in the decomposition chart is called column (row) multiplicity. 


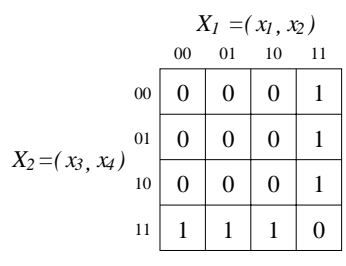

(a)

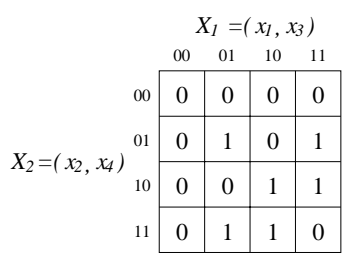

(b)
Figure 2.1: Decomposition chart.

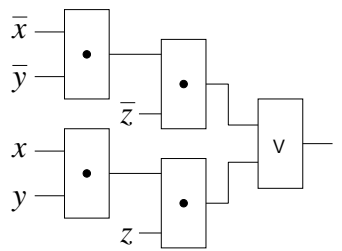

Figure 3.1: A realization of $f(x, y, z)=$ $\bar{x} \bar{y} \bar{z} \vee x y z$.

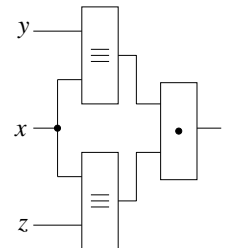

Figure 3.2: Non-disjoint bi-decomposition for $f(x, y, z)=\bar{x} \bar{y} \bar{z} \vee x y z$.
Example 2.2 In Fig. 2.1 (a), the row and column multiplicities are two. In Fig. 2.1 (b), the row and column multiplicities are four.

Definition 2.4 Let $\mu\left(f: X_{1}, X_{2}\right)$ be the column multiplicities for $f$ with respect to $X_{1}$ and $X_{2}$. Let $\mu(f$ : $\left.X_{2}, X_{1}\right)$ be the row multiplicities for $f$ with respect to $X_{1}$ and $X_{2}$.

Theorem 2.1 $f$ has a disjoint bi-decomposition of form $f\left(X_{1}, X_{2}\right)=h\left(g_{1}\left(X_{1}\right), g_{2}\left(X_{2}\right)\right)$ iff $\mu\left(f: X_{1}, X_{2}\right) \leq 2$ and $\mu\left(f: X_{2}, X_{1}\right) \leq 2$.

\section{Non-Disjoint Bi-Decomposition}

Definition 3.1 Let $X_{1}$ and $X_{2}$ be disjoint sets of variables, and let $x$ be disjoint from $X_{1}$ and $X_{2}$. A logic function $f$ has a non-disjoint bi-decomposition iff $f$ can be represented as $f\left(X_{1}, X_{2}, x\right)=h\left(g_{1}\left(X_{1}, x\right), g_{2}\left(X_{2}, x\right)\right)$, where $h$ is a two-variable logic function. In this case, $x$ is called the common variable.

A function $f$ with a non-disjoint bi-decomposition can be realized by the network shown in Fig. 1.3.

Lemma 3.1 Let $X=\left(X_{1}, X_{2}, x\right)$ be a partition of the input variables. Let $h\left(g_{1}, g_{2}\right)$ be an arbitrary logic function of two variables. Then,

$$
\begin{aligned}
& h\left(g_{1}\left(X_{1}, x\right), g_{2}\left(X_{2}, x\right)\right)= \\
& \quad \bar{x} h\left(g_{1}\left(X_{1}, 0\right), g_{2}\left(X_{2}, 0\right)\right) \vee x h\left(g_{1}\left(X_{1}, 1\right), g_{2}\left(X_{2}, 1\right)\right) .
\end{aligned}
$$

Definition 3.2 Let $x$ be the common variable of the non-disjoint bi-decomposition. Let $f\left(X_{1}, X_{2}, a\right)$ be a subfunction, where $x$ is set to a 0 or 1 .

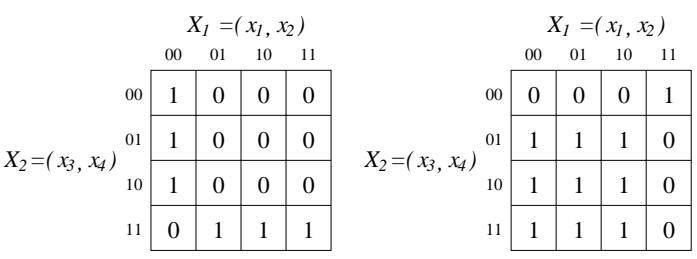

(a) $f_{0}=\bar{x}_{1} \bar{x}_{2} \oplus x_{3} x_{4}$

(b) $f_{1}=x_{1} x_{2} \oplus\left(x_{3} \vee x_{4}\right)$

Figure 3.3: Functions in Example 3.2

Theorem $3.1 f\left(X_{1}, X_{2}, x\right)$ has a non-disjoint bi-decomposition of the form $h\left(g_{1}\left(X_{1}, x\right), g_{2}\left(X_{2}, x\right)\right)$ iff $f\left(X_{1}, X_{2}, 0\right)$ and $f\left(X_{1}, X_{2}, 1\right)$ have disjoint bi-decompositions $h\left(g_{01}\left(X_{1}\right), g_{02}\left(X_{2}\right)\right)$ and $h\left(g_{11}\left(X_{1}\right), g_{12}\left(X_{2}\right)\right)$, respectively.

Example 3.1 Consider the three-variable function: $f(x, y, z)=\bar{x} \bar{y} \bar{z} \vee x y z$. Suppose modules that realizes any function of two variables can be used. The straightforward realization shown in Fig. 3.1 requires five modules. The Shannon expansion with respect to $x$ is $f(x, y, z)=\bar{x} f(0, y, z) \vee x f(1, y, z)$, where $f(0, y, z)=$ $\bar{y} \bar{z}$, and $f(1, y, z)=y z$. Note that both $f(0, y, z)$ and $f(1, y, z)$ have bi-decompositions with $h(x, y)=x y$. Since, $g_{1}(x, y)=\bar{x} g_{01}\left(X_{1}\right) \vee x g_{11}\left(X_{1}\right)=\bar{x} \bar{y} \vee x y$, and $g_{2}(x, y)=$ $\bar{x} g_{02}\left(X_{2}\right) \vee x g_{12}\left(X_{2}\right)=\bar{x} \bar{z} \vee x z$. We have $f(x, y, z)=$ $g_{1}(x, y) g_{2}(x, z)=(\bar{x} \bar{y} \vee x y)(\bar{x} \bar{z} \vee x z)$. From this expression, we have the network in Fig. 3.2. This network requires only three modules.

Example 3.2 Consider the five-variable function $f=$ $\bar{x}_{5} f_{0} \vee x_{5} f_{1}$, where $f_{0}$ and $f_{1}$ are shown in Fig. 3.3. Since both $f_{0}$ and $f_{1}$ have disjoint bi-decompositions of the form $h\left(g_{1}\left(X_{1}\right), g_{2}\left(X_{2}\right)\right), f=\bar{x}_{5} f_{0} \vee x_{5} f_{1}$ has a non-disjoint bidecomposition as follows:

$$
\begin{aligned}
f & =\bar{x}_{5}\left\{\bar{x}_{1} \bar{x}_{2} \oplus x_{3} x_{4}\right\} \vee x_{5}\left\{x_{1} x_{2} \oplus\left(x_{3} \vee x_{4}\right)\right\} \\
& =\left\{\bar{x}_{5}\left(\bar{x}_{1} \bar{x}_{2}\right) \vee x_{5}\left(x_{1} x_{2}\right)\right\} \oplus\left\{\bar{x}_{5}\left(x_{3} x_{4}\right) \vee x_{5}\left(x_{3} \vee x_{4}\right)\right\} .
\end{aligned}
$$

The converse is true also.

Up to now, we only considered the case where there is a single common variable. However, the theorem can be extened to $k$ common variables, where $k \geq 2$.

Definition 3.3 Let $X_{1}, X_{2}$, and $X_{3}$ be disjoint sets of variables. Let $f\left(X_{1}, X_{2}, a\right)$ be the sub-functions, where $X_{3}$ is set to $a \in\{0,1\}^{k}$, and $k$ denotes the number of variables in $X_{3}$.

Theorem 3.2 Let $X_{1}, X_{2}$, and $X_{3}$ be disjoint sets of variables. Then, $f$ has a non-disjoint bi-decomposition of form $f\left(X_{1}, X_{2}, X_{3}\right)=h\left(g_{1}\left(X_{1}, X_{3}\right), g_{2}\left(X_{2}, X_{3}\right)\right)$ iff $f\left(X_{1}, X_{2}, a\right)$ has a decomposition of the form $h\left(g_{1} \boldsymbol{a}\left(X_{1}\right), g_{2} \boldsymbol{a}\left(X_{2}\right)\right)$ for all possible $\boldsymbol{a} \in\{0,1\}^{k}$, where $k$ denotes the number of variables in $X_{3}$. 


\section{A Fast Method for Bi-Decompositions}

In this section, we show necessary and sufficient conditions for a function to have a disjoint bi-decomposition. Then, we show efficient algorithms to find disjoint bidecompositions. In the previous sections, $h\left(g_{1}, g_{2}\right)$ is an arbitrary two-variable logic function. To find a disjoint bi-decomposition, we need to consider only three types:

1) OR type: $f=g_{1}\left(X_{1}\right) \vee g_{2}\left(X_{2}\right)$,

2) AND type: $f=g_{1}\left(X_{1}\right) g_{2}\left(X_{2}\right)$, and

3) EXOR type: $f=g_{1}\left(X_{1}\right) \oplus g_{2}\left(X_{2}\right)$.

Since $f$ has an AND type disjoint bi-decomposition iff $\bar{f}$ has OR type disjoint bi-decomposition, we only consider the OR type and EXOR type bi-decompositions.

Definition $4.1 x$ and $\bar{x}$ are literals of a variable $x . A$ logical product which contains at most one literal for each variable is called a product term or a product. Product terms combined with OR operators form a sum-ofproducts expression (SOP).

Definition 4.2 A prime implicant (PI) p of a function $f$ is a product term which implies $f$, such that the deletion of any literal from $p$ results in a new product which does not imply $f$.

Definition 4.3 An irredundant sum-of-products expression (ISOP) is an SOP, where each product is a PI, and no product can be deleted without changing the function represented by the expression.

Definition 4.4 Let $f(X)$ be a function and $p$ be a product of literal $(s)$ in $X$. The restriction of $f$ to $p$, denoted by $f(X \mid p)$ is obtained as follows: If $x_{i}$ appears in $p$, then set $x_{i}$ in 1 in $f$, and if $\bar{x}_{i}$ appears in $p$, then set $x_{i}$ in 0 in $f$.

Example 4.1 Let $f\left(x_{1}, x_{2}, x_{3}\right)=x_{1} x_{2} \vee \bar{x}_{2} x_{3}$ and $p=$ $x_{1} x_{3} . f(X \mid p)$ is obtained as follows: Set $x_{1}=x_{3}=1$ in $f$, and we have $f\left(X \mid x_{1} x_{3}\right)=f\left(1, x_{2}, 1\right)=x_{2} \vee \bar{x}_{2}=1$.

Lemma $4.1 p$ is an implicant of $f(X)$, iff $f(X \mid p)=1$.

Example 4.2 By Lemma 4.1, $x_{1} x_{3}$ is an implicant of $x_{1} x_{2} \vee \bar{x}_{2} x_{3}$, shown in Example 4.1 .

Theorem 4.1 (OR type disjoint bi-decomposition) $f$ has a disjoint bi-decomposition of form $f\left(X_{1}, X_{2}\right)=g_{1}\left(X_{1}\right) \vee$ $g_{2}\left(X_{2}\right)$ iff every product in an ISOP for $f$ consists of literals from $X_{1}$ only or $X_{2}$ only.

Definition $4.5 x^{0}=\bar{x}, x^{1}=x$.

Corollary 4.1 If $f\left(x_{1}, x_{2}, \ldots, x_{n}\right)$ has a PI of the form $x_{1}^{a_{1}} x_{2}^{a_{2}} \cdots x_{n}^{a_{n}}$, where $a_{i} \in\{0,1\}$, then $f$ has no OR type disjoint bi-decomposition.
Let $x_{i}(i=1,2, \ldots, n)$ be the input variables of $f$. Let $p_{1} \vee$ $p_{2} \vee \cdots \vee p_{t}$ be an irredundant sum-of-products expression for $f$, where $p_{i}(i=1,2, \ldots, t)$ are PIs of $f$. Let $\Pi_{0}$ be the trivial partition of $\{1,2, \ldots, n\}, \Pi_{0}=[\{1\},\{2\}, \ldots,\{n\}]$.

Algorithm 4.1 (OR type disjoint bi-decomposition: $\left.f\left(X_{1}, X_{2}\right)=g_{1}\left(X_{1}\right) \vee g_{2}\left(X_{2}\right)\right)$.

1. For $i=1$ to $t$, form $\Pi_{i}$ from $\Pi_{i-1}$ by merging two blocks $\Omega_{1}$ and $\Omega_{2}$ of $\Pi_{i-1}$ if at least one literal in $p_{i}$ occurs in both $\Omega_{1}$ and $\Omega_{2}$.

2. If $\Pi_{t}$ has at least two blocks, then $f\left(X_{1}, X_{2}\right)$ has a disjoint bi-decomposition of the form $f\left(X_{1}, X_{2}\right)=$ $g_{1}\left(X_{1}\right) \vee g_{2}\left(X_{2}\right)$, with $X_{1}$ the union of one or more blocks of $\Pi_{t}$ and $X_{2}$ the union of the remaining blocks.

Example 4.3 Consider the ISOP: $f\left(x_{1}, x_{2}, \ldots, x_{6}\right)=$ $x_{1} x_{2} \vee x_{2} x_{3} \vee x_{4} x_{5} \vee x_{5} x_{6}$. The products $x_{1} x_{2}$ and $x_{2} x_{3}$ show that $x_{1}, x_{2}$, and $x_{3}$ are in the same block. Also, the products $x_{4} x_{5}$ and $x_{5} x_{6}$ show that $x_{4}, x_{5}$, and $x_{6}$ are in the same block. Thus, we have the partition $[\{1,2,3\},\{4,5,6\}]$. The corresponding OR type disjoint bi-decomposition is $f\left(X_{1}, X_{2}\right)=g_{1}\left(X_{1}\right) \vee g_{2}\left(X_{2}\right)$, where $X_{1}=\left(x_{1}, x_{2}, x_{3}\right)$ and $X_{2}=\left(x_{4}, x_{5}, x_{6}\right)$.

Example 4.4 Consider the function $f$ with an ISOP: $f\left(x_{1}, x_{2}, x_{3}, x_{4}, x_{5}\right)=x_{1} x_{2} x_{3} \vee x_{3} x_{4} x_{5}$.

1) The product $x_{1} x_{2} x_{3}$ shows that $x_{1}, x_{2}$, and $x_{3}$ belong to the same block.

2) The product $x_{3} x_{4} x_{5}$ shows that $x_{3}, x_{4}$, and $x_{5}$ belong to the same block.

Thus, all the variables belong to the same block. From this, it follows that $f$ has no OR type decomposition.

Theorem 4.2 (AND type disjoint bi-decomposition) $f$ has a disjoint bi-decomposition of form $f\left(X_{1}, X_{2}\right)=$ $g_{1}\left(X_{1}\right) g_{2}\left(X_{2}\right)$ iff every product in an ISOP for $\bar{f}$ consists of literals from $X_{1}$ only or $X_{2}$ only.

Lemma 4.2 [15] An arbitrary $n$-variable function can be uniquely represented as

$$
\begin{gathered}
f\left(x_{1}, x_{2}, \ldots, x_{n}\right)=a_{0} \oplus\left(a_{1} x_{1} \oplus a_{2} x_{2} \oplus \cdots \oplus a_{n} x_{n}\right) \\
\oplus\left(a_{12} x_{1} x_{2} \oplus a_{13} x_{1} x_{3} \oplus \cdots \oplus a_{n-1 n} x_{n-1} x_{n}\right) \\
\oplus \cdots \oplus a_{12 \cdots n} x_{1} x_{2} \cdots x_{n},
\end{gathered}
$$

where $a_{i} \in\{0,1\}$. The above expression is called a positive polarity Reed-Muller expression (PPRM).

For a given function $f$, the coefficients $a_{0}, a_{1}, a_{2}, \ldots, a_{12 \cdots n}$ are uniquely determined. Thus, the PPRM is a canonical representation. The number of products in (4.1) is at most $2^{n}$, and all the literals are positive (uncomplemented). 
Theorem 4.3 (EXOR type disjoint bi-decomposition) $f$ has a disjoint bi-decomposition of the form $f\left(X_{1}, X_{2}\right)=$ $g_{1}\left(X_{1}\right) \oplus g_{2}\left(X_{2}\right)$ iff every product in the PPRM for $f$ consists of literals from $X_{1}$ only or $X_{2}$ only.

Corollary 4.2 If the PPRM of an n-variable function has the product $x_{1} x_{2} \cdots x_{n}$, then $f$ has no EXOR type disjoint bi-decomposition.

Theorem 4.4 When $f$ has an EXOR type disjoint bidecomposition, the number of true minterms of $f$ is an even number.

Corollary 4.3 When the number of true minterms of $f$ is an odd number, then $f$ does not have an EXOR type disjoint bi-decomposition.

The significance of this observation is that at least one half of the functions can be quickly rejected as candidates for EXOR type disjoint bi-decomposition.

Let $x_{i}(i=1,2, \ldots, n)$ be the input variables of $f$. Let $p_{1} \oplus p_{2} \oplus \cdots \oplus p_{t}$ be PPRM for $f$, where $p_{i}(i=$ $1,2, \ldots, t)$ are products. Let, $\Pi_{0}$ be the trivial partition of $\{1,2, \ldots, n\}, \Pi_{0}=[\{1\},\{2\}, \ldots,\{n\}]$.

Algorithm 4.2 (EXOR type disjoint bi-decomposition: $\left.f\left(X_{1}, X_{2}\right)=g_{1}\left(X_{1}\right) \oplus g_{2}\left(X_{2}\right)\right)$.

1. For $i=1$ to $t$, form $\Pi_{i}$ from $\Pi_{i-1}$ by merging two blocks $\Omega_{1}$ and $\Omega_{2}$ of $\Pi_{i-1}$ if at least one literal in $p_{i}$ occurs in both $\Omega_{1}$ and $\Omega_{2}$.

2. If $\Pi_{t}$ has at least two blocks, then $f\left(X_{1}, X_{2}\right)$ has a disjoint bi-decomposition of form $f\left(X_{1}, X_{2}\right)=g_{1}\left(X_{1}\right) \oplus$ $g_{2}\left(X_{2}\right)$, with $X_{1}$ the union of one or more blocks of $\Pi_{t}$ and $X_{2}$ the union of the remaining blocks.

Example 4.5 Consider the PPRM: $f\left(x_{1}, x_{2}, \ldots, x_{6}\right)=$ $x_{1} x_{2} \oplus x_{2} x_{3} \oplus x_{4} x_{5} \oplus x_{5} x_{6}$. The products $x_{1} x_{2}$ and $x_{2} x_{3}$ show that $x_{1}, x_{2}$, and $x_{3}$ are in the same block. Also, the products $x_{4} x_{5}$ and $x_{5} x_{6}$ show that $x_{4}, x_{5}$, and $x_{6}$ are in the same block. Thus, we have the partition $[\{1,2,3\},\{4,5,6\}]$. The corresponding EXOR type disjoint bi-decomposition is $f\left(X_{1}, X_{2}\right)=g_{1}\left(X_{1}\right) \oplus g_{2}\left(X_{2}\right)$, where $X_{1}=\left(x_{1}, x_{2}, x_{3}\right)$ and $X_{2}=\left(x_{4}, x_{5}, x_{6}\right)$.

Algorithm 4.3 (Non-disjoint bi-decomposition). $f\left(X_{1}, X_{2}, x_{i}\right)=g_{1}\left(X_{1}, x_{i}\right) \otimes g_{2}\left(X_{2}, x_{i}\right)$, where $\otimes$ denotes either $O R, A N D$, or EXOR. Let $\left(X_{1}, X_{2}, x_{i}\right)$ be a partition of the variables $x_{1}, x_{2}, \ldots$, and $x_{n}$. For $i=1$ to $n$, do

i) Let $f_{0 i}=f\left(X_{1}, X_{2}, 0\right) .\left(\right.$ Set $x_{i}$ to 0$)$. Let $f_{1 i}=$ $f\left(X_{1}, X_{2}, 1\right)$. (Set $x_{i}$ to 1$)$.

ii) If both $f_{0 i}$ and $f_{1 i}$ have the same type of disjoint bidecompositions with the same partition, then $f$ has a non-disjoint bi-decomposition.

\section{Complexity Analysis of the Algorithms}

\subsection{OR type disjoint bi-decomposition}

We assume that the function is given as an ISOP with $t$ products. Note that $t \leq 2^{n-1}$. The time to form the partition of variables is $O(n \cdot t)$.

\subsection{EXOR type disjoint bi-decomposition}

A PPRM can be represented by a functional decision diagram (FDD [5, 15]). Each path from the root node to the constant 1 node corresponds to a product in the PPRM. Thus, the partition of the input variables is directly generated from the FDD. The number of paths in an FDD is $O\left(2^{n}\right)$, where $n$ is the number of the input variables. However, we can avoid exhaustive generation of paths as follows: Let $p_{1}$ and $p_{2}$ be products in a PPRM. If all the literals in $p_{1}$ also appear in $p_{2}$, then $p_{2}$ need not be generated in the Algorithm, since the product $p_{1}$ that contains more literals than $p_{2}$ is more important. By searching the paths with more literals first, we can efficiently detect functions with no disjoint bi-decomposition.

Example 5.1 Consider the function $f(X)$ given as a PPRM: $f(X)=x_{1} \oplus x_{1} x_{2} \oplus x_{3} x_{4} \oplus x_{1} x_{2} x_{5} x_{6}$. In constructing the partition of $X$, we need not consider the products $x_{1}$ or $x_{1} x_{2}$, since $x_{1} x_{2} x_{5} x_{6}$ has the literals of $x_{1}$ and $x_{1} x_{2}$. In this case, the product $x_{1} x_{2} x_{5} x_{6}$ shows that $x_{1}, x_{2}, x_{5}$, and $x_{6}$ belong to the same group. Also, the product $x_{3} x_{4}$ shows that $x_{3}$ and $x_{4}$ belong to the same group. Thus, $X$ is partitioned as $X=\left(X_{1}, X_{2}\right)$, where $X_{1}=\left(x_{1}, x_{2}, x_{5}, x_{6}\right)$ and $X_{2}=\left(x_{3}, x_{4}\right)$.

Definition 5.1 Let $p$ be a product. The set of variables in $p$ is denoted by $V(p)=\left\{x_{i} \mid x_{i}\right.$ or $\bar{x}_{i}$ appears in $\left.p\right\}$. For example, $V\left(x_{1} x_{2} \bar{x}_{4}\right)=\left\{x_{1}, x_{2}, x_{4}\right\}$

Definition 5.2 Let $F$ be a PPRM. A product $p$ is said to have maximal variable set $V(p)$ if there is no other product $p^{\prime}$ such that $V(p) \subset V\left(p^{\prime}\right)$.

Example 5.2 For the PPRM, F $=x_{1} x_{2} \oplus x_{1} x_{3} \oplus$ $x_{1} x_{2} x_{3} \oplus x_{4}, \quad V\left(x_{1} x_{2}\right)=\left\{x_{1}, x_{2}\right\}, V\left(x_{1} x_{3}\right)=$ $\left\{x_{1}, x_{3}\right\}, V\left(x_{1} x_{2} x_{3}\right)=\left\{x_{1}, x_{2}, x_{3}\right\}$, and $V\left(x_{4}\right)=\left\{x_{4}\right\}$. Thus, $x_{1} x_{2} x_{3}$ and $x_{4}$ have maximal variable sets.

Theorem 5.1 A function $f$ has an EXOR type disjoint bi-decomposition if a function $f^{\prime}$ from the PPRM of $f$ by eliminating implicants not having maximal variable sets has an EXOR type disjoint bi-decomposition.

The following theorem says that if a function has an EXOR type disjoint bi-decomposition, then the number of products in the PPRM is relatively small.

Theorem 5.2 If $f$ has a disjoint bi-decomposition of the form $f\left(X_{1}, X_{2}\right)=g_{1}\left(X_{1}\right) \oplus g_{2}\left(X_{2}\right)$, then the number of products in the PPRM is at most $2^{n_{1}}+2^{n_{2}}-1$, where $n_{i}$ is the number of variables in $X_{i}(i=1,2)$. 


\section{Number of Functions with Bi-Decompositions}

\subsection{Functions with a small number of variables}

In the previous sections, we showed that disjoint bidecompositions are easy to find. In this section, we will enumerate the functions with disjoint bi-decompositions.

Definition 6.1 A function $f$ is said to be nondegenerate if for all $x_{i} f\left(\mid \bar{x}_{i}\right) \neq f\left(\mid x_{i}\right)$.

Definition 6.2 Two functions $f$ and $g$ are NPequivalent, denoted by $f \stackrel{\mathrm{NP}}{\sim} g$, iff $g$ is derived from $f$ by the following operations:

1) Permutation of the input variables.

2) Negations of the input variables.

The following is easy to prove.

Lemma 6.1 If $f$ has a disjoint bi-decomposition and if $f \stackrel{\mathrm{NP}}{\sim} g$, then $g$ has also the same type of disjoint bidecomposition.

Lemma 6.2 All the two-variable functions have disjoint bi-decompositions.

Example 6.1 There are $2^{2^{3}}=256$ three-variable logic functions of which 218 are nondegenerate. These nondegenerate functions are grouped into $16 \mathrm{NP}$-equivalence classes as shown in Table 6.1 [9]. In this table, the column headed by $N$ denotes the number of functions in that equivalence class. Eight classes have disjoint bi-decompositions, and three have non-disjoint bi-decompositions. Note that 194 functions have bidecompositions.

The number of functions with AND type disjoint bidecompositions is equal to the number of functions with OR type disjoint bi-decompositions.

In the case of disjoint bi-decompositions, a function has exactly one type of decomposition (Lemma 6.4). On the other hand, in the case of non-disjoint bidecompositions, a function may have more than one type of bi-decompositions.

Example 6.2 Consider the three-variable function $f=$ $\bar{x}_{1} x_{3} \vee x_{1} x_{2}$. This function has three types of non-disjoint bi-decompositions:

$$
\begin{aligned}
f & =\bar{x}_{1} x_{3} \vee x_{1} x_{2} & & \text { (OR type bi-decomposition) } \\
& =\bar{x}_{1} x_{3} \oplus x_{1} x_{2} & & \text { (EXOR type bi-decomposition) } \\
& =\left(x_{1} \vee x_{3}\right)\left(\bar{x}_{1} \vee x_{2}\right) & & \text { (AND type bi-decomposition) }
\end{aligned}
$$

Table 6.1: NP-representative functions of three variables.

\begin{tabular}{|r|l|r|l|l|}
\hline & Representative functions & $N$ & Type & Property \\
\hline 1 & $x_{1} \oplus x_{2} \oplus x_{3}$ & 2 & EXOR & \\
2 & $x_{1} x_{2} x_{3}$ & 8 & AND & Disjoint \\
3 & $x_{1} \vee x_{2} \vee x_{3}$ & 8 & OR & Bi-Decomposition \\
4 & $x_{1}\left(x_{2} \vee x_{3}\right)$ & 24 & AND & \\
5 & $x_{1} \vee x_{2} x_{3}$ & 24 & OR & \\
6 & $x_{1}\left(x_{2} \oplus x_{3}\right)$ & 12 & AND & \\
7 & $x_{1} \vee\left(x_{2} \oplus x_{3}\right)$ & 12 & OR & \\
8 & $x_{1} \oplus x_{2} x_{3}$ & 24 & EXOR & \\
\hline 9 & $x_{1} x_{2} x_{3} \vee \bar{x}_{1} \bar{x}_{2} \bar{x}_{3}$ & 4 & & \\
10 & $\left(x_{1} \vee x_{2} \vee x_{3}\right)\left(\bar{x}_{1} \vee \bar{x}_{2} \vee \bar{x}_{3}\right)$ & 4 & & Non-Disjoint \\
11 & $\bar{x}_{1} x_{3} \vee x_{1} x_{2}$ & 24 & & Bi-Decomposition \\
12 & $x_{1} \bar{x}_{2} \bar{x}_{3} \vee x_{2} x_{3}$ & 24 & & \\
13 & $\left(x_{1} \vee \bar{x}_{2} \vee \bar{x}_{3}\right)\left(x_{2} \vee x_{3}\right)$ & 24 & & \\
\hline 14 & $x_{1} x_{2} \vee x_{2} x_{3} \vee x_{3} x_{1}$ & 8 & & No \\
15 & $x_{1} x_{2} \vee x_{2} x_{3} \vee x_{1} x_{3} \vee \bar{x}_{1} \bar{x}_{2} \bar{x}_{3}$ & 8 & & Bi-Decomposition \\
16 & $\bar{x}_{1} x_{2} x_{3} \vee x_{1} \bar{x}_{2} x_{3} \vee x_{1} x_{2} \bar{x}_{3}$ & 8 & & \\
\hline
\end{tabular}

\begin{tabular}{|c|c|c|c|c|c|}
\hline & $n=2$ & $n=3$ & $n=4$ \\
\hline \multicolumn{3}{|l|}{ All the functions } & 16 & 256 & 65536 \\
\hline \multicolumn{3}{|c|}{ Nondegenerate functions } & 10 & 218 & 64594 \\
\hline \multirow{5}{*}{$\begin{array}{l}\text { Functions with } \\
\text { bi-decomposition }\end{array}$} & Disjoint & $\mathrm{AND}$ & 4 & 44 & 1660 \\
\hline & & OR & 4 & 44 & 1660 \\
\hline & & EXOR & 2 & 26 & 914 \\
\hline & \multicolumn{2}{|c|}{ Non-disjont } & 0 & 80 & 3860 \\
\hline & \multicolumn{2}{|l|}{ Total } & 10 & 194 & 8094 \\
\hline
\end{tabular}

$N$ : Number of the functions in the class.

Table 6.2: Number of functions.

\subsection{The number of functions with} bi-decompositions

Lemma 6.3 [4]: Let $\alpha(n)$ be the number of nondegenerate functions on $n$ variables. Then,

$$
\alpha(n)=\sum_{k=0}^{n}\left(\begin{array}{l}
n \\
k
\end{array}\right)(-1)^{n-k} 2^{2^{k}} \sim 2^{2^{n}},
$$

where $a(n) \sim b(n)$ means $\lim _{n \rightarrow \infty} \frac{a(n)}{b(n)}=1$.

Lemma 6.4 A nondegenerate function $f$ has at most one type of disjoint bi-decomposition:

1. $f\left(X_{1}, X_{2}\right)=g_{1}\left(X_{1}\right) \cdot g_{2}\left(X_{2}\right)$,

2. $f\left(X_{1}, X_{2}\right)=g_{1}\left(X_{1}\right) \vee g_{2}\left(X_{2}\right)$, or

3. $f\left(X_{1}, X_{2}\right)=g_{1}\left(X_{1}\right) \oplus g_{2}\left(X_{2}\right)$,

where $g_{1}$ and $g_{2}$ are nondegenerate functions on one or more variables.

Theorem 6.1 The number of functions $N_{\text {disjoint }}(n)$ with disjoint bi-decompositions is $N_{\text {disjoint }}(n)=A_{\text {dis }}(n)+$ $O_{\text {dis }}(n)+E_{\text {dis }}(n)$, where

$$
A_{\text {dis }}(n)=n ! \sum_{\substack{k_{1}, k_{2}, \ldots, k_{n} \geq 0 \\ 1 k_{1}+2 k_{2}+\cdots+n k_{n}=n}} \prod_{i=1}^{n}\left(\frac{\alpha(i)-A_{\text {dis }}(i)}{i !}\right)^{k_{i}} \frac{1}{k_{i} !}
$$


Table 7.1: Number of functions with bi-decompositions.

\begin{tabular}{|l|l|r|}
\hline Decomposition Type & Number of Functions \\
\hline \multirow{4}{*}{ Disjoint } & AND & 853 \\
\cline { 2 - 3 } & OR & 264 \\
\cline { 2 - 3 } & EXOR & 73 \\
\hline \multirow{4}{*}{ Non-disjoint } & AND & 162 \\
\cline { 2 - 3 } & OR & 91 \\
\cline { 2 - 3 } & EXOR & 42 \\
\hline
\end{tabular}

$$
\begin{aligned}
& O_{d i s}(n)=n ! \sum_{\substack{k_{1}, k_{2}, \ldots, k_{n} \geq 0 \\
1 k_{1}+2 k_{2}+\cdots+n k_{n}=n}} \prod_{i=1}^{n}\left(\frac{\alpha(i)-O_{\text {dis }}(i)}{i !}\right)^{k_{i}} \frac{1}{k_{i} !} \\
& E_{d i s}(n)=2 n ! \sum_{\substack{k_{1}, k_{2}, \ldots, k_{n} \geq 0 \\
1 k_{1}+2 k_{2}+\cdots+n k_{n}=n}} \prod_{i=1}^{n}\left(\frac{\alpha(i)-E_{d i s}(i)}{i !}\right)^{k_{i}} \frac{1}{2^{k_{i} k_{i} !}}
\end{aligned}
$$

where the sums are over all partitions of $n$ except the trivial partition $n=0 \cdot 1+0 \cdot 2+\cdots+0 \cdot(n-1)+1 \cdot n$ (i.e. the sum is over all partitions where $k_{n}=0$ ), and where $A_{\text {dis }}(1)=O_{\text {dis }}(1)=E_{\text {dis }}(1)=0$.

Table 6.2 shows the number of functions with disjoint bidecompositions up to $n=4$.

\section{Experimental Results}

We analyzed the bi-decomposability of 136 benchmark functions. Over these multiple-output functions, the total number of outputs (functions) is 1908. For each function, we determined whether there exists a disjoint bidecomposition. If none existed, we determined if there exists a non-disjoint bi-decomposition (with a single common variable). Table 7.1 summarizes our results. It is interesting that 1190 out of 1908 functions, or 62 percent, have disjoint bi-decompositions. Of the remaining 718 functions, 295 have non-disjoint decompositions. It should be noted that more than 295 functions have non-disjoint decompositions, since a function with a disjoint bi-decomposition may also have a non-disjoint bidecomposition.

\section{Conclusions and Comments}

In this paper, we presented the bi-decomposition, a special case of functional decomposition. Disjoint bidecompositions have the following features:

1) They are easy to detect; we use ISOPs or PPRMs rather than decomposition charts.

2) Programmable logic devices exist that realize bidecompositions.

3) If the function has an OR (AND) type bi-decomposition, then we can optimize the expression separately.

We enumerated functions with bi-decompositions. Among 218 nondegenerate functions of 4 variables, 194 have bi- decompositions. Also, we derived formulae for the number of disjoint bi-decompositions.

Since the fraction of functions with decompositions approaches to zero as $n$ increase [4], the fraction of functions with bi-decompositions also approaches to zero as $n$ increases. However, for 1908 functions we analyzed about $78 \%$ of them had either disjoint or non-disjoint bidecompositions.

\section{References}

[1] R. L. Ashenhurst, "The decomposition of switching functions," In Proceedings of an International Symposium on the Theory of Switching, pp. 74-116, April 1957.

[2] J. T. Butler, "On the number of functions realized by cascades and disjunctive networks," IEEE Trans. Comput., Vol. C-24, pp. 681-690, July 1975.

[3] H. A. Curtis, A New Approach to the Design of Switching Circuits, Princeton, N.J.: Van Nostrand, 1962.

[4] M. A. Harrison, Introduction to Switching and Automata Theory, McGraw-Hill, 1965.

[5] U. Kebschull, E. Schubert and W. Rosenstiel, "Multilevel logic synthesis based on functional decision diagrams," EDAC 9\%, 1992, pp. 43-47.

[6] Y-T. Lai, M. Pedram, S. B. K. Vrudhula, "EVBDD-based algorithm for integer linear programming, spectral transformation, and functional decomposition," IEEE Trans. $C A D$, Vol. 13, No. 8. Aug. 1994, pp. 959-975.

[7] A. A. Malik, D. Harrison, and R. K. Brayton, "Threelevel decomposition with applicatioin to PLDs," ICCD1991, pp. 628-633, Oct. 1991.

[8] Y. Matsunaga, "An attempt to factor logic functions using exclusive-or decomposition," SASIMI'96, pp. 78-83.

[9] S. Muroga, Logic Design and Switching Theory, John Wiley \& Sons, 1979.

[10] T. Sasao and K. Kinoshita, "On the number of fanoutfree functions and unate cascade functions," IEEE Trans. on Comput., Vol. C-28, No. 1, pp. 866-72, Jan. 1979.

[11] T. Sasao, "Input variable assignment and output phase optimization of PLA's," IEEE Trans. Comput., Vol. C33, No. 10, pp. 879-894, Oct. 1984.

[12] T. Sasao, "Application of multiple-valued logic to a serial decomposition of PLA's," International Symposium on Multiple-Valued Logic, Guangzhou, China, pp. 264-271, May 1989.

[13] T. Sasao (ed.), Logic Synthesis and Optimization, Kluwer Academic Publishers, 1993.

[14] T. Sasao, "A design method for AND-OR-EXOR three-level networks," $A C M / I E E E$ International Workshop on Logic Synthesis, Tahoe City, California, May 2326, 1995, pp.8:11- 8:20.

[15] T. Sasao and M. Fujita (ed.), Representations of Discrete Functions, Kluwer Academic Publishers, 1996.

[16] T. Sasao and J. T. Butler, "Comparison of the worst and best sum-of-products expressions for multiple-valued functions," (preprint).

[17] M. Sauerhoff, I. Wegener, and R. Werchner, "Optimal ordered binary decision diagrams for tree-like circuits," SASIMI'96 (to be published).

[18] S. Yang, "Logic synthesis and optimization benchmark user guide, version 3.0," $M C N C$, Jan. 1991. 